\title{
BOUNDS ON THE NUMBER OF PRIMES IN RAMANUJAN INTERVAL
}

\author{
JAN FELIKSIAK
}

\begin{abstract}
\end{abstract}
The Ramanujan primes are the least positive integers $\mathcal{R}_{n}$ having the property that if $m \geq \mathcal{R}_{n}$, then $\pi_{m}-\pi_{(m / 2)} \geq n$. This document develops several bounds related to the Ramanujan primes, sharpening the currently known results. The theory presented is by no means exhaustive, however it provides insights for future research work. Alternatively, we may say that it is a road map which may be followed to make further discoveries.

2010 Mathematics Subject Classification. 0102, 1102, 1103, 11A25, 11A41, 11 N05.

Key words and phrases. Prime counting function Supremum/Infimum, prime numbers distribution, Ramanujan Interval, Ramanujan primes. 


\section{Contents}

Acknowledgement

1. Bounds on the Number of Primes on the Ramanujan Interval 3

1.1. Bounds on $s$ and $p_{s}$ on the Ramanujan Interval 3

2. Tailored Log Integral Bounds on $\pi_{\left(\mathcal{R}_{n}\right)} \quad 4$

2.1. Upper/Lower Bounds on $\pi_{\left(\mathcal{R}_{n}\right)}=s \quad 5$

3. Bounds on $\pi_{\left(\mathcal{R}_{n}\right)}$ in terms of the ordinal number $n \quad 7$

4. Bounds on $p_{s}$ on Ramanujan Interval 10

5. Alternative Bound on $\mathcal{R}_{n}$ on the Ramanujan Interval 13

References 


\section{ACKNOWLEDGEMENT}

I am very grateful to Dr. John W. Nicholson, for providing hints and directions by the means of his own research papers and internet communications, pertinent to the area of this research.

\section{J. Feliksiak}

\section{Bounds on the Number of Primes}

Ramanujan primes term was first used by Sondow [16], who mentions: "I coined the term Ramanujan primes in 2005 ... Subsequently, I came across a completely different meaning of the term". It originated with the Theorem of Ramanujan:

Theorem 1.1 (Ramanujan).

Let $\pi_{m}$ denote the number of primes not exceeding $m \in \mathbb{N}$. Then, $\pi_{m}-\pi_{(m / 2)} \geq 1,2,3,4,5, \ldots$ if $m \geq 2,11,17,29,41, \ldots$ respectively.

The interval satisfying this condition will be referred to as the Ramanujan interval. The definition of the Ramanujan prime follows:

Definition 1.2 (Ramanujan prime).

For $n \geq 1$ the $\mathrm{n}$-th Ramanujan prime is the least positive integer $\mathcal{R}_{n}$ having the property that if $m \geq \mathcal{R}_{n}$, then $\pi_{m}-\pi_{(m / 2)} \geq n$.

Note that $\mathcal{R}_{n}$ is indeed a prime, by minimality condition the function $\pi_{m}-\pi_{(m / 2)}$ and $\pi_{m}$ must increase at $m=\mathcal{R}_{n}$. Since they can increase by at most 1 , the equality $\pi_{\mathcal{R}_{n}}-\pi_{\left(\mathcal{R}_{n} / 2\right)}=n$ holds (Sondow [16]).

\subsection{Bounds on $s$ and $p_{s}$ on the Ramanujan Interval.}

From the paper under the title "An improved upper bound for Ramanujan primes" by Srinivasan et al [19], we have the upper bound on $s \in \mathbb{N}$ :

Theorem 1.3 (Srinivasan et al: Theorem 1).

Let $\mathcal{R}_{n}=p_{s}$ be the $n$-th Ramanujan prime, where $p_{s}$ is the s-th prime. Then, for all $n \in \mathbb{N} \mid n \geq 19, \mathcal{R}_{19}=227$ :

$$
s<2 n\left(1+\frac{3}{\log (n)+\log (\log n)-4}\right)
$$

\section{Remark 1.1.}

The function in Theorem 1.3, has a vertical asymptote between $n=18$ and $n=$ 19. The difference of $\left(2 n\left(1+\frac{3}{\log (n)+\log (\log n)-4}\right)\right)-s$, at $n=18$ attains approx. -2244.67 , while at $n=19$ it attains approx. 4669.33. Please refer to Figure 1 . The difference, is greater than zero for all $n \geq 19, \mathcal{R}_{19}=227$, with a global minimum occurring at $n=50, \mathcal{R}_{50}=641$, where it attains approx. 219.095. 

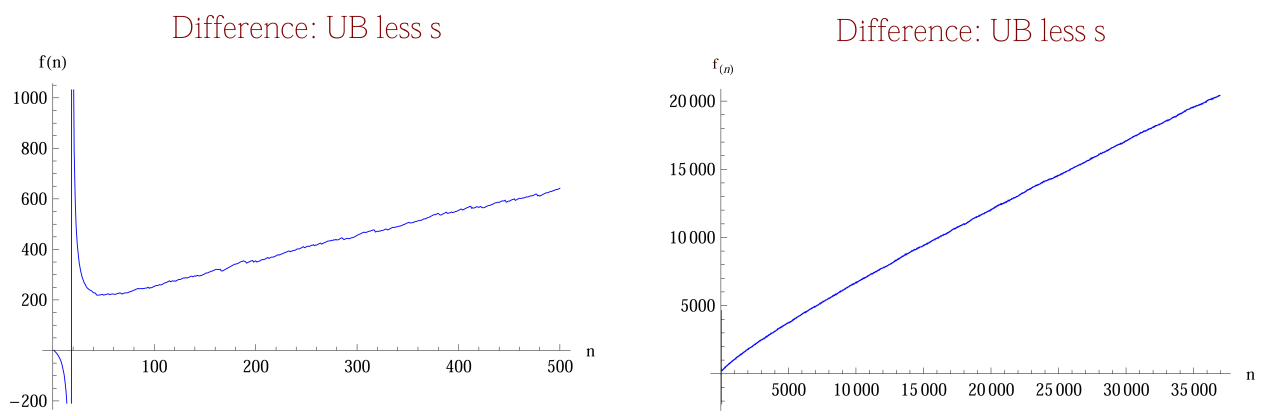

Figure 1. The drawing shows the difference of the function $2 n\left(1+\frac{3}{\log (n)+\log (\log n)-4}\right)$ and $s$. The asymptote is clearly visible between $n=18$ and $n=19$, the global minimum occurs at $n=50$ where it attains approx. 219.095. The figure is drawn at every $n \in \mathbb{N}$ in the range.

2. Tailored Log Integral Bounds ON $\pi_{\left(\mathcal{R}_{n}\right)}$

\section{Bounds on prime counting function}

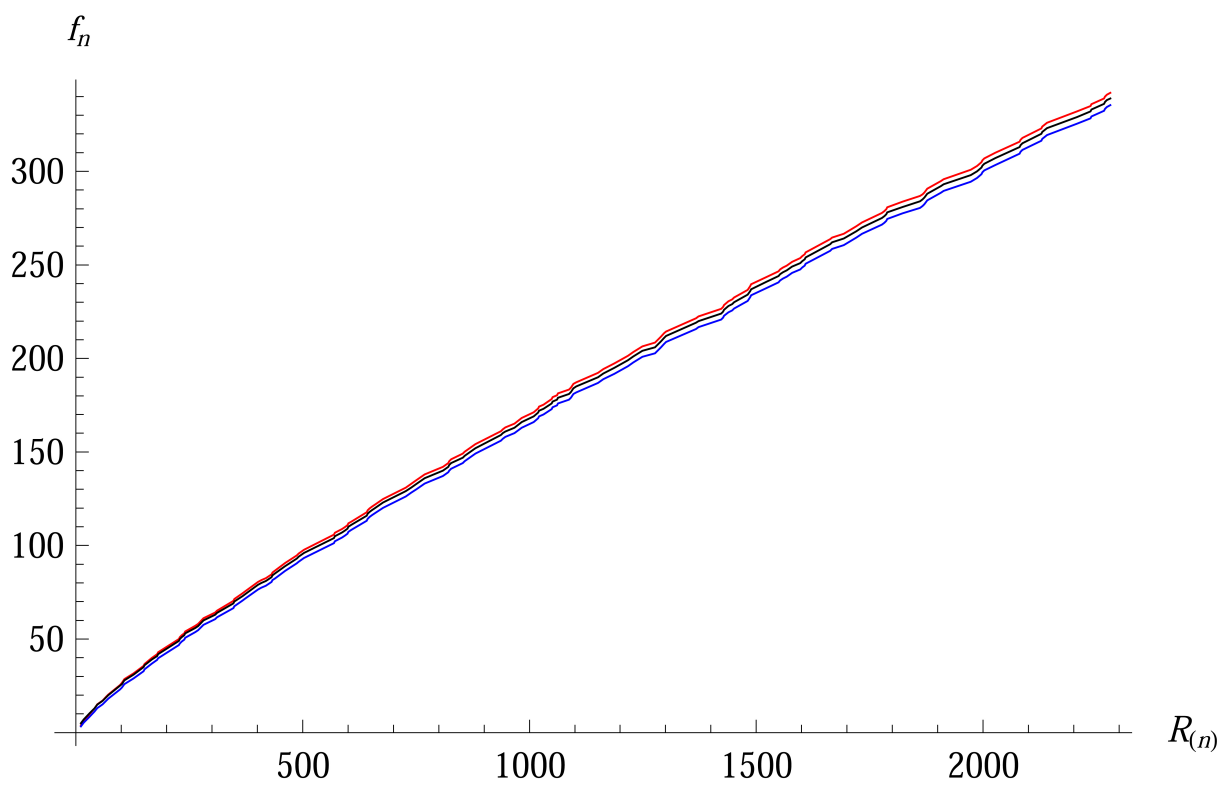

Figure 2. The drawing shows the Supremum (Red) and Infimum (Blue) bounds on $\pi_{\mathcal{R}_{n}}$ (Black). The Figure is drawn at every $\mathcal{R}_{n}$ in the range $2 \leq \mathcal{R}_{n} \leq 2281$.

In this document we present improved bounds pertaining to the case $\mathcal{R}_{n}=p_{s}$, where $n, s \in \mathbb{N}$ are ordinal numbers indicating the $n^{\text {th }}$ Ramanujan prime, and $s^{t h}$ 

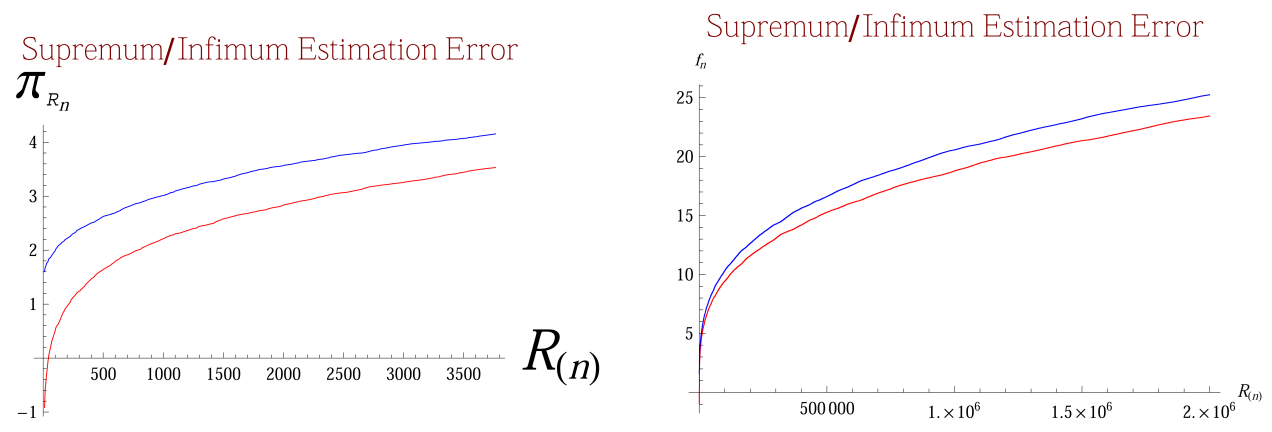

FIGURE 3. The drawing shows the estimation error made by the Supremum (Red) and Infimum (Blue) of the prime counting function $\pi_{\mathcal{R}_{n}}$. The figure is drawn at every $\mathcal{R}_{n} \in \mathbb{N}$ in the range.

prime. Thus we have that in the case $\mathcal{R}_{n}=p_{s}$, necessarily $s=\pi_{\left(\mathcal{R}_{n}\right)}$. In such a case we can improve the estimate of $s$ very significantly by implementing the Tailored integral theorems by Feliksiak [3], which provide sharper estimates. The Tailored logarithmic integral definitions follow:

Definition 2.1 (Supremum Upper integration limit).

$$
\theta_{\mathcal{S}}=\log p_{\left(\mathcal{R}_{n}\right)} \sharp=\sum_{p \leq \mathcal{R}_{n}} \log p \quad \text { where } p \text { is the prime } p \in \mathbb{N} \mid p \leq \mathcal{R}_{n}
$$

Definition 2.2 (Infimum Upper integration limit).

$$
\theta_{\mathcal{I}}=\sum_{p \leq \mathcal{R}_{n}}\left((\log (p))-\frac{\pi(\log \log p)}{(p)^{\gamma}}\right) \text { where } p \text { is the prime } p \in \mathbb{N} \mid p \leq \mathcal{R}_{n}
$$

and $\gamma$ is the Euler-Mascheroni constant $\gamma \approx 0.57721566490153286060651209$.

Theorem 2.3 (Supremum and Infimum Bound on s).

Let $\mathcal{R}_{n}=p_{s}$ be the $n^{\text {th }}$ Ramanujan prime, where $p_{s}$ is the $s^{\text {th }}$ prime. Then, for all $n \in \mathbb{N} \mid n \geq 47$ :

$\operatorname{Inf}_{\left(\mathcal{R}_{n}\right)}=\int_{2}^{\theta_{\mathcal{I}}} \frac{d t}{\log t}<\pi_{\mathcal{R}_{n}}=s<\int_{2}^{\theta_{\mathcal{S}}} \frac{d t}{\log t}=\operatorname{Sup}_{\left(\mathcal{R}_{n}\right)} \quad \forall \mathcal{R}_{n} \in \mathbb{N} \mid \mathcal{R}_{n} \geq \mathcal{R}_{6}=47$

Proof.

For the proof, please refer to Feliksiak [3].

\subsection{Upper/Lower Bounds on $\pi_{\left(\mathcal{R}_{n}\right)}=s$.}

The bounds given by Theorem 2.3, are computationally quite demanding for larger $\mathcal{R}_{n} \in \mathbb{N}$. Consequently more efficient bounds are given by Theorem 2.6: 


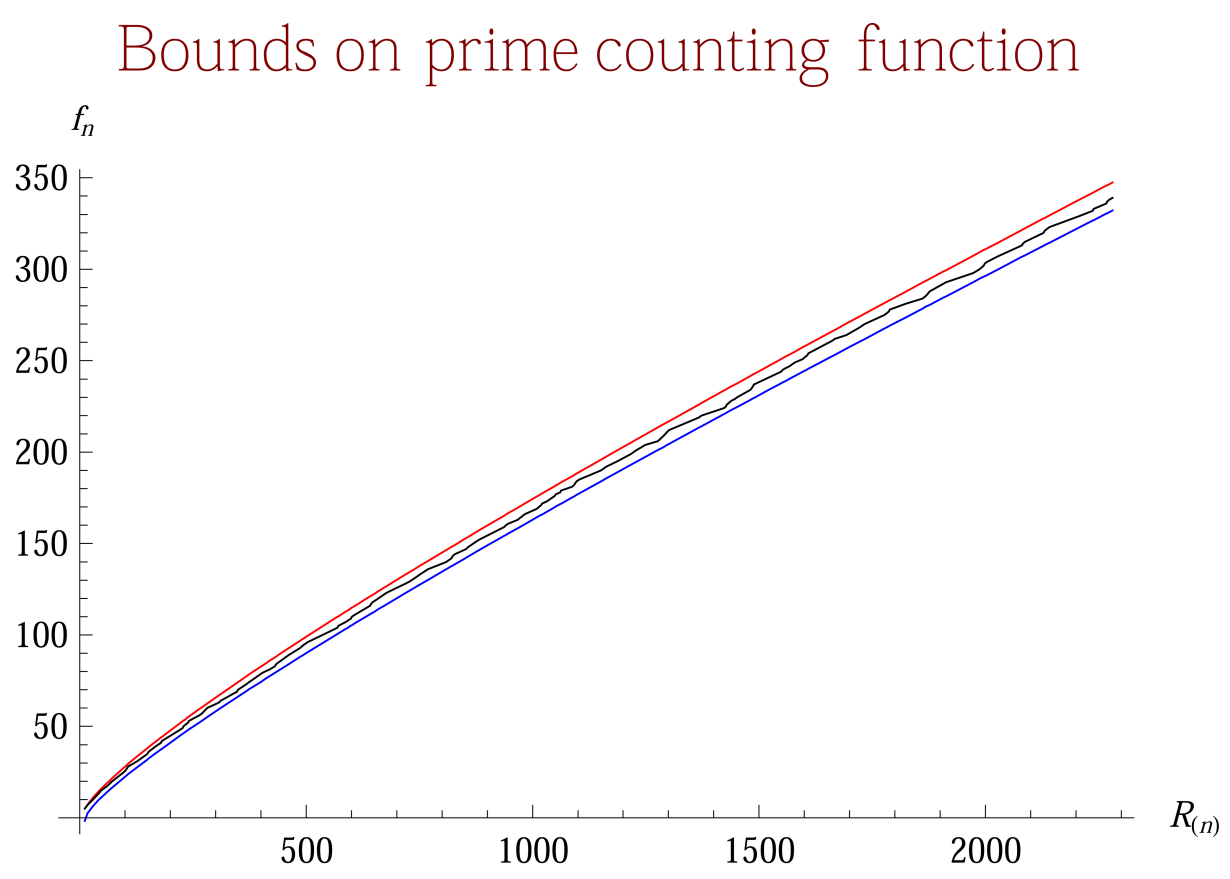

FiguRE 4. The drawing shows the Upper (Red) and Lower (Blue) bounds on $\pi_{\mathcal{R}_{n}}=s$ (Black). The Figure is drawn at every $\mathcal{R}_{n}$ in the range $2 \leq \mathcal{R}_{n} \leq 2281$.
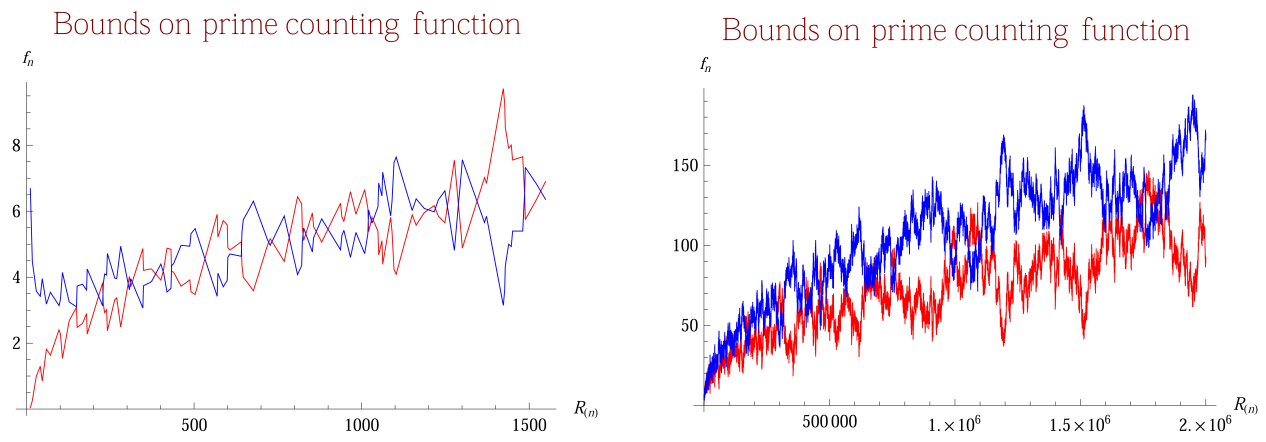

Figure 5. The drawing shows the estimation error made by the Upper (Red) and Lower (Blue) Bounds of the prime counting function $\pi_{\mathcal{R}_{n}}=s$. The R.H. figure is drawn at every $n \in \mathbb{N}$ in the range $2 \leq n \leq 70434$.

Definition 2.4 (Upper Bound integration limit).

$$
\begin{array}{r}
\theta_{\mathcal{U}}=\left(\mathcal{R}_{(n)}-(\sqrt{5}-1)\left(4 \gamma^{2}-2 \gamma\right)\left(\log \mathcal{R}_{(n)}\right) \sqrt[3]{\mathcal{R}_{(n)}}\right) \\
\text { where the prime } \mathcal{R} \in \mathbb{N} \mid \mathcal{R}_{n} \geq 47
\end{array}
$$


Definition 2.5 (Lower Bound integration limit).

$$
\theta_{\mathcal{L}}=\mathcal{R}_{(n)}-\left(\exp \left(\gamma+\frac{1}{2}\right)\right) \sqrt{\left(\mathcal{R}_{n}\right)} \quad \text { where } p \text { is the prime } p \in \mathbb{N} \mid p \leq \mathcal{R}_{n}
$$

Theorem 2.6 (Upper and Lower Bounds on s).

Let $\mathcal{R}_{n}=p_{s}$ be the $n^{\text {th }}$ Ramanujan prime, where $p_{s}$ is the $s^{\text {th }}$ prime. Then, for all $n \in \mathbb{N} \mid n \geq 47$ :

$L B_{\left(\mathcal{R}_{n}\right)}=\int_{2}^{\theta_{\mathcal{L}}} \frac{d t}{\log t}<\pi_{\mathcal{R}_{n}}=s<\int_{2}^{\theta_{\mathcal{U}}} \frac{d t}{\log t}=U B_{\left(\mathcal{R}_{n}\right)} \quad \forall \mathcal{R}_{n} \in \mathbb{N} \mid \mathcal{R}_{n} \geq \mathcal{R}_{6}=47$

Proof.

For the proof, please refer to Feliksiak [3].

3. BOUNDS ON $\pi_{\left(\mathcal{R}_{n}\right)}$ IN TERMS OF THE ORDINAL NUMBER $n$
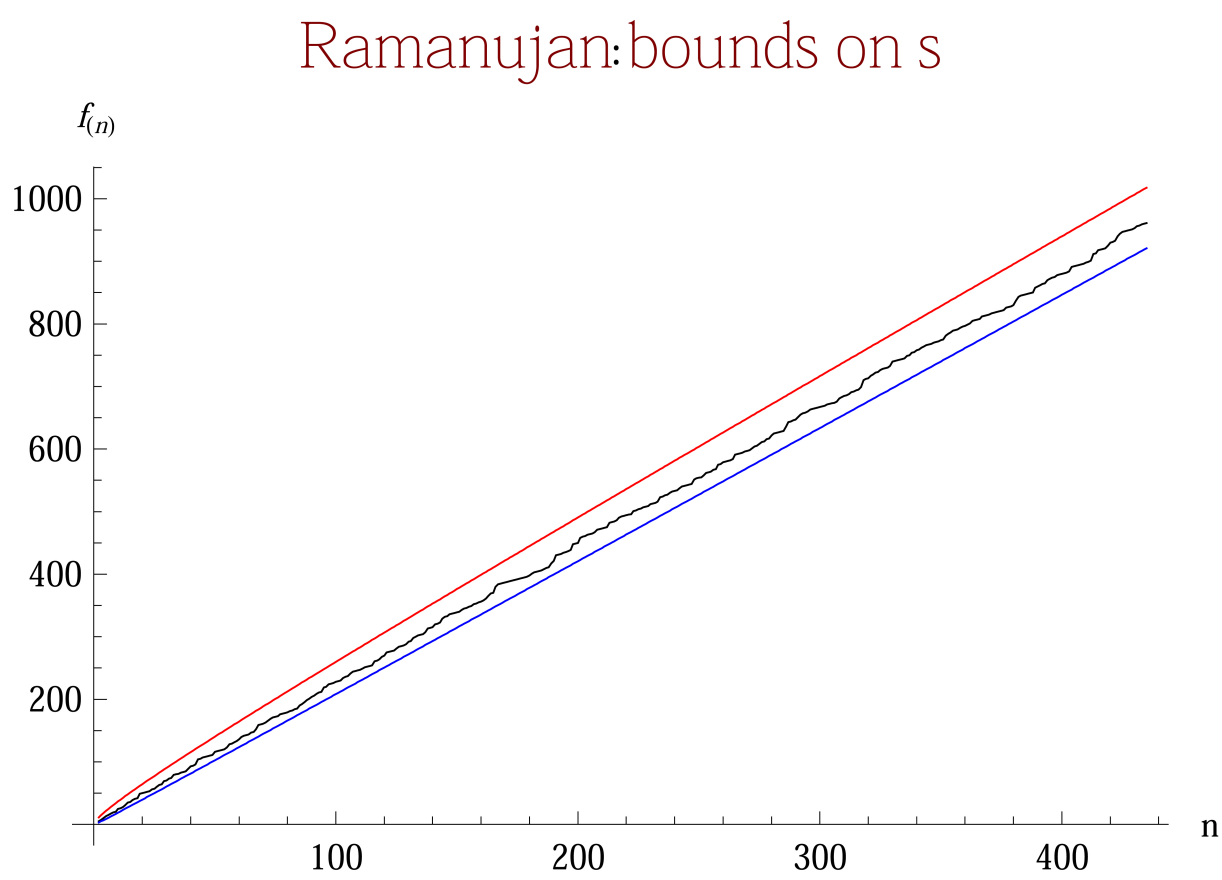

Figure 6. The drawing shows the Upper (Red) and Lower (Blue) bounds on $\pi_{\mathcal{R}_{n}}=s$ (Black). The Figure is drawn at every $n$ in the range $2 \leq n \leq 435$.

There may be the need to express $\pi_{\left(\mathcal{R}_{n}\right)}=s$ in terms of the ordinal number $n$. Therefore, elementary upper and lower bounds on $s$ in terms of the ordinal number $n$ are given by Theorem 3.1 (please also refer to Figures 6 and 7). 

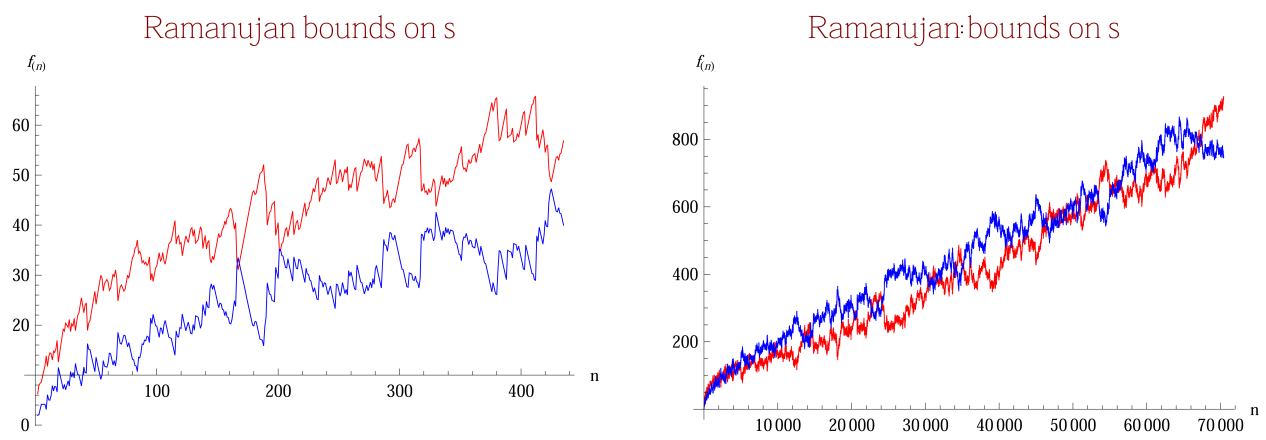

Figure 7. The drawing shows the estimation error made by the Upper (Red) and Lower (Blue) Bounds of the prime counting function $\pi_{\mathcal{R}_{n}}=s$. The R.H. figure is drawn at every $n \in \mathbb{N}$ in the range $2 \leq n \leq 70434$.

Theorem 3.1 (Upper and Lower Bounds).

The bounds on $\pi_{\mathcal{R}_{n}}=s$, s.t. $s \in \mathbb{N}$ subject to $p_{s}=\mathcal{R}_{n}$, in terms of the ordinal number $n$ for all $n \in \mathbb{N} \mid n \geq 2$ is given by:

$$
\mathcal{L B}_{n}=\sqrt{5}(n)^{\alpha}-\sqrt{n} \leq \pi_{\left(\mathcal{R}_{n}\right)} \leq 2.09(n)^{\beta}+5 \sqrt{n}=\mathcal{U} \mathcal{B}_{n}
$$

Where $p_{s}$ is the $s^{\text {th }}$ prime number s.t. $\forall n \in \mathbb{N} \mid p_{s}=\mathcal{R}_{n}$ and $R_{n}$ is given by the Definition 1.2 and the exponents are:

$$
\alpha=\frac{9947}{10000} \text { and } \beta=\frac{10008}{10000}
$$

The proof is limited to the proof of the Upper Bound only. The proof of the Lower Bound is left at the discretion of the reader.

Proof.

Suppose that the Upper Bound of Theorem 3.1 is false for $n \in \mathbb{N} \mid n \geq 20000$. Then necessarily,

$$
\mathcal{U} \mathcal{B}_{n}-\pi_{\left(\mathcal{R}_{n}\right)}=2.09(n)^{\beta}+5 \sqrt{n}-\pi_{\left(\mathcal{R}_{n}\right)}<0
$$

Thus clearly,

$$
2.09(n)^{\beta}+4 \sqrt{n}-\pi_{\left(\mathcal{R}_{n}\right)}<2.09(n)^{\beta}+5 \sqrt{n}-\pi_{\left(\mathcal{R}_{n}\right)}<0
$$

The Cauchy Root test of the absolute value of the difference 3.3 for $n \in \mathbb{N} \mid n \geq$ 20000 is:

$$
\sqrt[n]{\left|a_{n}\right|}=\sqrt[n]{\left|2.09(n)^{\beta}+4 \sqrt{n}-\pi_{\left(\mathcal{R}_{n}\right)}\right|}>1
$$

At $n=20000$ the root test 3.4 attains approximately 1.00023 and tends to 1 strictly from above. By the definition of the Cauchy Root test therefore, the sequence is diverging. In accordance with the hypothesis therefore, the difference 3.3 must diverge to $-\infty$ as $n$ increases unboundedly. However, at $n=20000$, the difference 3.3 attains approximately 99.1734 and diverges at an increasing rate as $n$ increases unboundedly. Consequently, we have a contradiction to the initial 
hypothesis. Thus the difference 3.2 for $n \in \mathbb{N} \mid n \geq 20000$ exceeds at every step $\sqrt{n}$.

Necessarily, it must be true that the UB of Theorem 3.1 is true for $n \in \mathbb{N} \mid n \geq$ 20000. It is a straightforward exercise to verify that UB of Theorem 3.1 holds within the range $2 \leq n \leq 20000$ as well, please refer to Figure 8 .

This implies that the Upper Bound of Theorem 3.1 holds for all $n \in \mathbb{N} \mid n \geq 2$, consequently this concludes the proof.
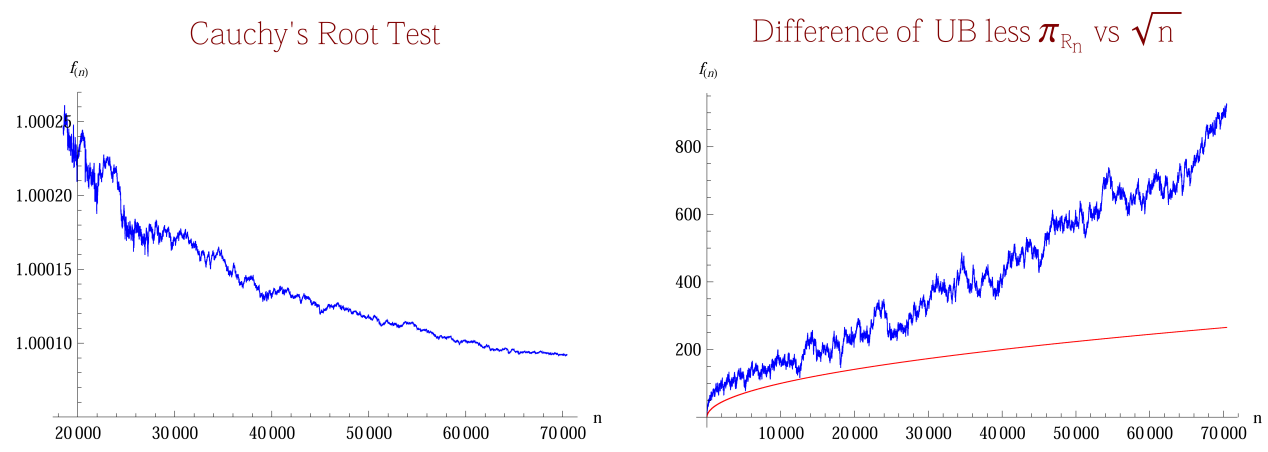

FiguRE 8. The L.H. drawing shows the Cauchy's Root Test, equation 3.4. The R.H. drawing compares the estimation error made by the Upper Bound on $\pi_{\left(\mathcal{R}_{n}\right)}$ vs $\sqrt{n}$ function. The R.H. figure is drawn at every $n \in \mathbb{N}$ in the range $2 \leq n \leq 70435$. 
4. Bounds ON $p_{s}$ ON RAMANUJAN INTERVAL

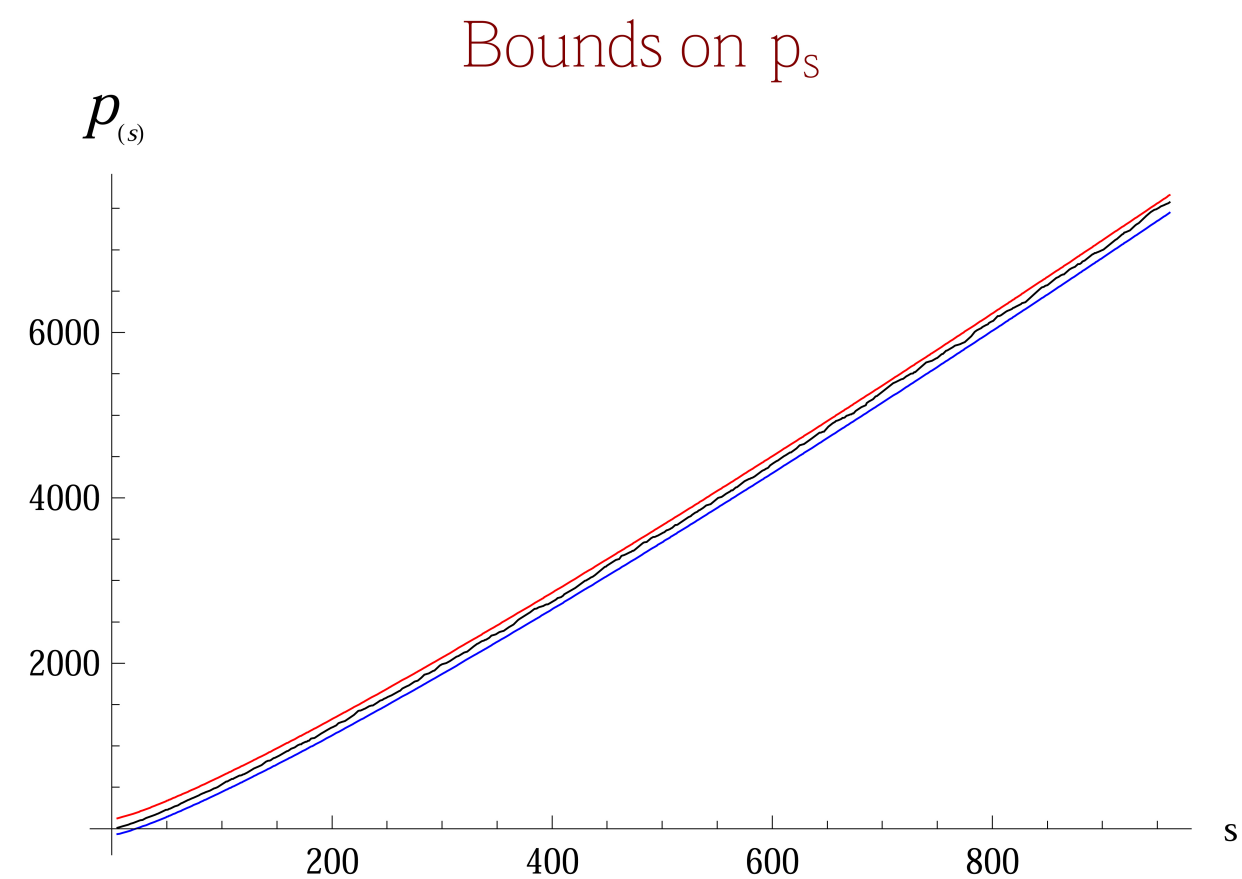

FiguRE 9. The drawing shows the Upper (Red) and Lower (Blue) bounds on $p_{s}$ (Black). The Figure is drawn at every $s$ in the range $5 \leq s \leq 961$.
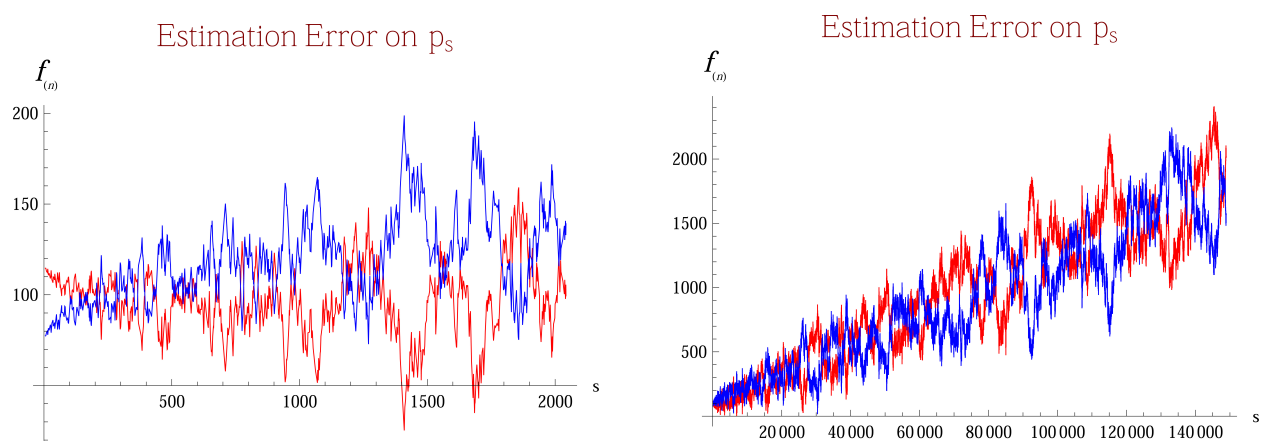

FiguRE 10. The drawing shows the estimation error made by the Upper (Red) and Lower (Blue) Bounds on the prime $p_{s}$. The R.H. figure is drawn at every $s \in \mathbb{N}$ in the range $5 \leq s \leq 148933$. 
Theorem 4.1 (Elementary Bounds on $p_{s}$ ).

The upper bound on the prime number $p_{s}$, where $p_{s}$ denotes the sequential $s^{\text {th }}$ prime number, for all $n, s \in \mathbb{N} \mid p_{s}=\mathcal{R}_{n} \geq 11$ is given by:

$$
\begin{aligned}
\mathcal{L B}_{s}=\left(s\left(\log (s)+\log \log s-\frac{97}{100}\right)-72\right)<p_{s} \\
\quad<\left(s\left(\log (s)+\log \log s-\frac{9472}{10000}\right)+120\right)=\mathcal{U B}_{s}
\end{aligned}
$$

The proof is limited to the proof of the Lower Bound only. The analogous proof of the Upper Bound is left at the discretion of the reader.

Proof.

We begin with:

$$
p_{(s)} \geq \mathcal{L B}_{s}
$$

Suppose that Theorem 4.1 is false for some $p_{s} \in \mathbb{N} \mid p_{s} \geq 151$, in accordance with the hypothesis this implies that:

$$
\exp \left(p_{(s)}\right)-\exp \left(\mathcal{L B}_{s}\right)<0
$$

However, at $p_{s}=151$ the difference 4.3 attains approximately $3.7885 \times 10^{65}$ and further increases exponentially. Therefore, we apply the d'Alemberts Ratio Test. Define a sequence for all prime numbers $p_{(s-1)}, p_{(s)} \in \mathbb{N} \mid p_{(s)} \geq 151$ :

Definition 4.2. $a_{(s)}=\frac{\exp \left(p_{(s)}\right)-\exp \left(\mathcal{L B}_{s}\right)}{\exp \left(p_{(s-1)}\right)-\exp \left(\mathcal{L B}_{(s-1)}\right)}$

Remark 4.1. The sequence $a_{(s)}$ given by the Definition 4.2 , has the least value at the Ramanujan twin primes, since the difference $p_{(s)}-p_{(s-1)}=2$. Consequently, it is therefore both necessary and sufficient, to consider the sequence $a_{(s)}$ at the Ramanujan twin primes only, with $p_{(s)}=6 i+7 \mid i \in \mathbb{N}, i \geq 24$.

\section{At the Ramanujan twin primes:}

$$
\exp \left(p_{(s)}\right)=\exp \left(p_{(s-1)}+2\right)=\exp \left(p_{(s-1)}\right) \times \exp (2)
$$

Thus, at the Ramanujan twin primes the sequence $a_{(s)}$ equals:

$$
a_{(s)}=\frac{\exp \left(p_{(s)}\right)-\exp \left(\mathcal{L B}_{s}\right)}{\exp \left(p_{(s-1)}\right)-\exp \left(\mathcal{L B}_{(s-1)}\right)}=\exp (2) \times\left[\frac{\exp \left(p_{(s-1)}\right)-\frac{\exp \left(\mathcal{L B}_{s}\right)}{\exp (2)}}{\exp \left(p_{(s-1)}\right)-\exp \left(\mathcal{L} \mathcal{B}_{(s-1)}\right)}\right]
$$

The bracketed expression on the RHS, at the Ramanujan twin primes approaches the limit:

$$
\lim _{n \rightarrow \infty}\left[\frac{\exp \left(p_{(s-1)}\right)-\frac{\exp \left(\mathcal{L B}_{s}\right)}{\exp (2)}}{\exp \left(p_{(s-1)}\right)-\exp \left(\mathcal{L B}_{(s-1)}\right)}\right] \rightarrow 1
$$

Therefore, at the Ramanujan twin primes, the sequence $a_{(s)}$ must clearly approach the limit:

$$
\lim _{n \rightarrow \infty} a_{(s)}=\lim _{n \rightarrow \infty}\left[\exp (2) \times\left[\frac{\exp \left(p_{(s-1)}\right)-\frac{\exp \left(\mathcal{L B}_{s}\right)}{\exp (2)}}{\exp \left(p_{(s-1)}\right)-\exp \left(\mathcal{L B}_{(s-1)}\right)}\right]\right] \rightarrow \exp (2)
$$


By the definition of the d'Alemberts Ratio Test therefore, the difference exp $\left(p_{(s)}\right)$ $\exp \mathcal{L B}_{s}$, diverges as $p_{(s)}$ increases unboundedly. Thus, it logically follows that at Ramanujan twin primes:

$$
\exp \left(\mathcal{L B}_{s}\right) \leq \exp \left(p_{(s)}\right) \quad \forall p_{(s)} \in \mathbb{N} \mid p_{(s)} \geq 151
$$

Necessarily therefore, we have a contradiction to the initial hypothesis. Since at the Ramanujan twin primes the sequence $\exp \left(p_{(s)}\right)-\exp \mathcal{L B}_{s}$ approaches:

$$
\left[\exp \left(p_{(s)}\right)-\exp \left(\mathcal{L B}_{s}\right)\right] \sim \exp (2)\left[\exp \left(p_{(s-1)}\right)-\exp \left(\mathcal{L B}_{(s-1)}\right)\right]
$$

Rearranging the above, we obtain that at the Ramanujan twin primes $\exp \mathcal{L B}_{s}$ approaches:

$$
\exp \left(\mathcal{L B}_{s}\right) \sim \exp \left(p_{(s)}\right)-\exp (2)\left[\exp \left(p_{(s-1)}\right)-\exp \left(\mathcal{L B}_{(s-1)}\right)\right]
$$

This in turn implies that a strict inequality holds:

$$
\exp \left(\mathcal{L B}_{s}\right)<\exp \left(p_{(s)}\right)
$$

Since increasing the gap between the consecutive primes has the effect of exponentially increasing the value that the sequence $a_{(s)}$ attains, therefore this result holds for all $p_{(s)} \in \mathbb{N} \mid p_{(s)} \geq 151$. By taking the logarithms of both sides, we obtain:

$$
\mathcal{L B}_{s}<p_{(s)} \quad \forall p_{(s)} \in \mathbb{N} \mid p_{(n)} \geq 151
$$

Thus, Theorem 4.1 holds for all $p_{(s)} \in \mathbb{N} \mid p_{(s)} \geq 151$. Direct computation verifies that Theorem 4.1 holds for all $p_{(s)} \in \mathbb{N} \mid 11 \leq p_{(s)} \leq 151$. Please refer to Figures 9 and 10. Therefore, Theorem 4.1 holds as stated:

$$
\mathcal{L B}_{s}<p_{(s)} \quad \forall s \in \mathbb{N} \mid s \geq 5
$$

Concluding the proof of Theorem 4.1. 
5. Alternative Bound on $\mathcal{R}_{n}$ ON the Ramanujan Interval

This section develops a new set of bounds on $\mathcal{R}_{n}$ w.r.t. $n \in \mathbb{N}$ in the Ramanujan Interval. These bounds constitute a significant improvement upon previously known bounds. The initial estimate presented by J. Sondow [16] had been further researched and optimized, producing bounds that follow $\mathcal{R}_{n}$ quite closely.

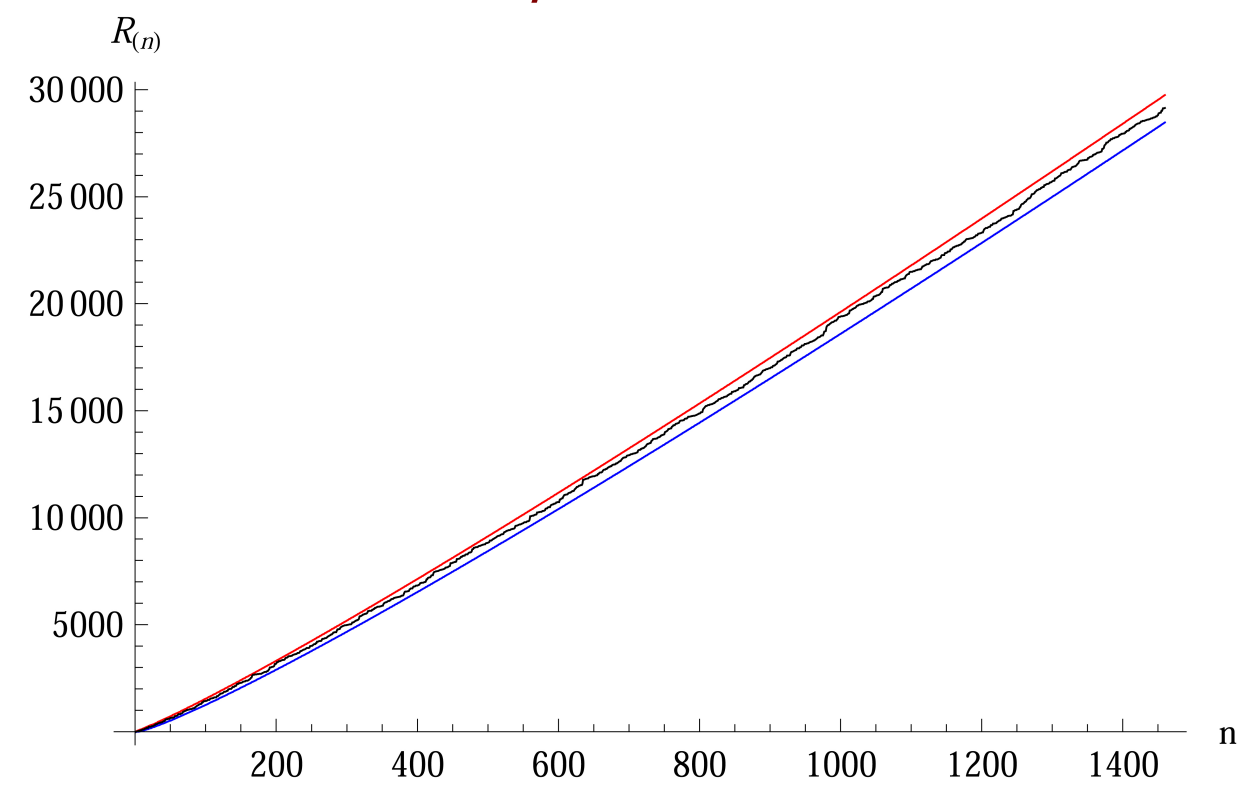

Figure 11. The drawing shows the Upper (Red) and Lower (Blue) bounds on $\mathcal{R}_{n}$ (Black). The Figure is drawn at every $n$ in the range $1 \leq n \leq 1460$.

Theorem 5.1 (Bounds on $\mathcal{R}_{n}$, w.r.t. $n$ in the Ramanujan Interval).

The upper and lower bounds on the Ramanujan prime number $\mathcal{R}_{n}$, w.r.t. the ordinal number $n$, which denotes both the sequential $n$ - th Ramanujan prime number $\mathcal{R}_{n}$ and the number of primes contained within the Ramanujan Interval $\left\{\frac{n}{2}, n\right\}$, are given by:

$$
\begin{array}{ll}
\mathcal{L B}_{n}=\left(n \sqrt{2 \pi}(\log (4 n))^{\alpha}-10 \sqrt{2 n}\right) \leq \mathcal{R}_{n} & \forall n \in \mathbb{N} \mid n \geq 1, \mathcal{R}_{n} \geq 2 \\
\mathcal{R}_{n} \leq\left(n \sqrt{2 \pi}(\log (4 n))^{\beta}+10 \sqrt{2 n}\right)=\mathcal{U} \mathcal{B}_{n} \quad \forall n \in \mathbb{N} \mid n \geq 1, \mathcal{R}_{n} \geq 2
\end{array}
$$

With $\alpha$ given by $\alpha=\frac{599}{625}$ and $\beta$ is given by $\beta=\frac{601}{625}$.

The proof is limited to the proof of the Lower Bound only. The proof of the Upper Bound is left at the discretion of the reader. 

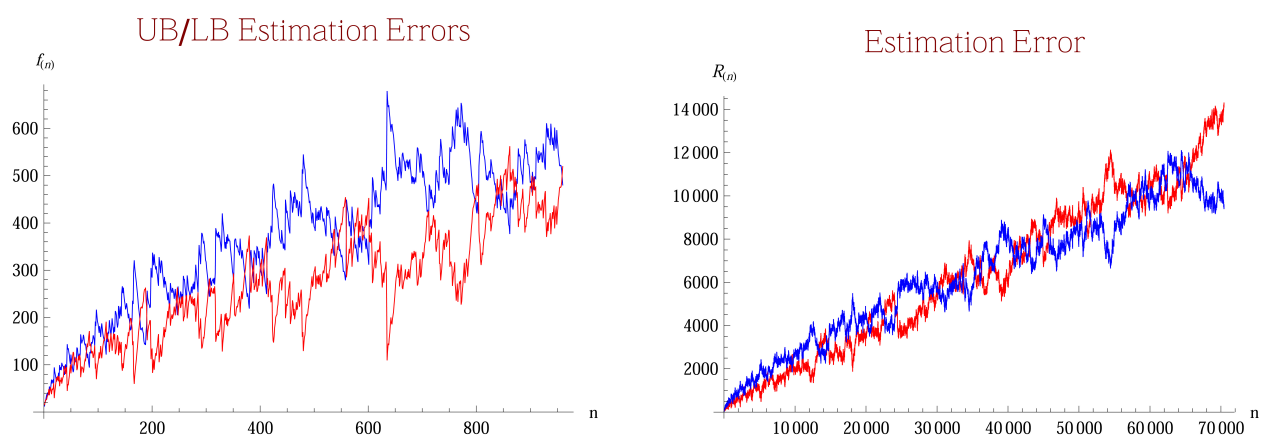

FiguRE 12. The drawing shows the estimation error made by the Upper (Red) and Lower (Blue) Bounds on $\mathcal{R}_{n}$. The R.H. figure is drawn at every $n \in \mathbb{N}$ in the range $1 \leq n \leq 70435$.

Proof.

We begin with:

$$
\mathcal{R}_{n} \geq \mathcal{L B}_{n}
$$

Suppose that Theorem 5.1 is false for some $\mathcal{R}_{n} \in \mathbb{N} \mid \mathcal{R}_{n} \geq 151$. In accordance with the hypothesis this implies that:

$$
\exp \left(\mathcal{R}_{n}\right)-\exp \left(\mathcal{L B}_{n}\right)<0
$$

However, at $\mathcal{R}_{n}=151$ the difference 5.4 attains approximately $1.70404 \times 10^{993}$ and further increases exponentially. Therefore, we apply the d'Alemberts Ratio Test. Define a sequence for all prime numbers $\mathcal{R}_{(n-1)}, \mathcal{R}_{n} \in \mathbb{N} \mid \mathcal{R}_{n} \geq 151$ :

Definition 5.2. $a_{(n)}=\frac{\exp \left(\mathcal{R}_{n}\right)-\exp \left(\mathcal{L B}_{n}\right)}{\exp \left(\mathcal{R}_{(n-1)}\right)-\exp (\mathcal{L B}(n-1))}$

Remark 5.1. The sequence $a_{(n)}$ given by the Definition 5.2, has the least value at the Ramanujan twin primes, since the difference $\mathcal{R}_{(n)}-\mathcal{R}_{(n-1)}=2$. Consequently, it is therefore both necessary and sufficient, to consider the sequence $a_{(n)}$ at the Ramanujan twin primes only, with $\mathcal{R}_{(n)}=6 i+7 \mid i \in \mathbb{N}, i \geq 24$.

At the Ramanujan twin primes:

$$
\exp \left(\mathcal{R}_{(n)}\right)=\exp \left(\mathcal{R}_{(n-1)}+2\right)=\exp \left(\mathcal{R}_{(n-1)}\right) \times \exp (2)
$$

Thus, at the Ramanujan twin primes the sequence $a_{(n)}$ equals:

$a_{(n)}=\frac{\exp \left(\mathcal{R}_{(n)}\right)-\exp \left(\mathcal{L B}_{n}\right)}{\exp \left(\mathcal{R}_{(n-1)}\right)-\exp \left(\mathcal{L B}_{(n-1)}\right)}=\exp (2) \times\left[\frac{\exp \left(\mathcal{R}_{(n-1)}\right)-\frac{\exp \left(\mathcal{L B}_{n}\right)}{\exp (2)}}{\exp \left(\mathcal{R}_{(n-1)}\right)-\exp \left(\mathcal{L B}_{(n-1)}\right)}\right]$

The bracketed expression on the RHS, at the Ramanujan twin primes approaches the limit:

$$
\lim _{n \rightarrow \infty}\left[\frac{\exp \left(\mathcal{R}_{(n-1)}\right)-\frac{\exp \left(\mathcal{L B}_{n}\right)}{\exp (2)}}{\exp \left(\mathcal{R}_{(n-1)}\right)-\exp \left(\mathcal{L B} \mathcal{B}_{(n-1)}\right)}\right] \rightarrow 1
$$


Therefore, at the Ramanujan twin primes, the sequence $a_{(n)}$ must clearly approach the limit:

$$
\lim _{n \rightarrow \infty} a_{(n)}=\lim _{n \rightarrow \infty}\left[\exp (2) \times\left[\frac{\exp \left(\mathcal{R}_{(n-1)}\right)-\frac{\exp \left(\mathcal{L B}_{n}\right)}{\exp (2)}}{\exp \left(\mathcal{R}_{(n-1)}\right)-\exp \left(\mathcal{L B}_{(n-1)}\right)}\right]\right] \rightarrow \exp (2)
$$

By the definition of the d'Alemberts Ratio Test therefore, the difference exp $\left(\mathcal{R}_{(n)}\right)-$ $\exp \mathcal{L B}_{n}$, diverges as $\mathcal{R}_{(n)}$ increases unboundedly. Thus, it logically follows that at Ramanujan twin primes:

$$
\exp \left(\mathcal{L B}_{n}\right) \leq \exp \left(\mathcal{R}_{(n)}\right) \quad \forall \mathcal{R}_{(n)} \in \mathbb{N} \mid \mathcal{R}_{(n)} \geq 151
$$

Necessarily therefore, we have a contradiction to the initial hypothesis. Since at the Ramanujan twin primes the sequence $\exp \left(\mathcal{R}_{(n)}\right)-\exp \mathcal{L B}_{n}$ approaches:

$$
\left[\exp \left(\mathcal{R}_{(n)}\right)-\exp \left(\mathcal{L B}_{n}\right)\right] \sim \exp (2)\left[\exp \left(\mathcal{R}_{(n-1)}\right)-\exp \left(\mathcal{L B}_{(n-1)}\right)\right]
$$

Rearranging the above, we obtain that at the Ramanujan twin primes $\exp \mathcal{L B}_{n}$ approaches:

$$
\exp \left(\mathcal{L B}_{n}\right) \sim \exp \left(\mathcal{R}_{(n)}\right)-\exp (2)\left[\exp \left(\mathcal{R}_{(n-1)}\right)-\exp \left(\mathcal{L B}_{(n-1)}\right)\right]
$$

This in turn implies that a strict inequality holds:

$$
\exp \left(\mathcal{L B}_{n}\right)<\exp \left(\mathcal{R}_{(n)}\right)
$$

Since increasing the gap between the consecutive primes has the effect of exponentially increasing the value that the sequence $a_{(n)}$ attains, therefore this result holds for all $\mathcal{R}_{(n)} \in \mathbb{N} \mid \mathcal{R}_{(n)} \geq 151$. By taking the logarithms of both sides, we obtain:

$$
\mathcal{L B}_{n}<\mathcal{R}_{(n)} \quad \forall \mathcal{R}_{(n)} \in \mathbb{N} \mid \mathcal{R}_{(n)} \geq 151
$$

Thus, Theorem 5.1 holds for all $\mathcal{R}_{(n)} \in \mathbb{N} \mid \mathcal{R}_{(n)} \geq 151$. Direct computation verifies that Theorem 5.1 holds for all $\mathcal{R}_{(n)} \in \mathbb{N} \mid 11 \leq \mathcal{R}_{(n)} \leq 151$. Please refer to Figures 11 and 12. Therefore, Theorem 5.1 holds as stated:

$$
\mathcal{L B}_{n}<\mathcal{R}_{(n)} \quad \forall n \in \mathbb{N} \mid n \geq 2
$$

Concluding the proof of Theorem 5.1 .

\section{Remark 5.2.}

This remark provides a few hints for the proof of the Upper Bound on $\mathcal{R}_{(n)}$ : Equation 5.2. It can be used to further sharpen the Upper Bound, and help to facilitate an enhancement of the Lower bound in a similar fashion. Observe, that the corresponding ratio:

$$
\frac{1}{\exp (2)} \times\left[\frac{\exp \left(\mathcal{U B}_{n}\right)-\exp \left(\mathcal{R}_{(n)}\right)}{\exp \left(\mathcal{U} \mathcal{B}_{(n-1)}\right)-\exp \left(\mathcal{R}_{(n-1)}\right)}\right]
$$

at $\mathcal{R}_{n} \geq 11$ it exceeds:

$$
(2 \pi)(4 n)^{\alpha}+10 n^{\beta}
$$

With the exponents given by:

$$
\alpha=\left(\left(\frac{\sqrt{5}+1}{2}\right)+0.5222\right) \quad \beta=\left(\frac{\sqrt{5}+1}{2}\right)
$$


Please also refer to Figures 13 and 14. Hence, the limit diverges:

$$
\lim _{n \rightarrow \infty} a_{(n)}=\lim _{n \rightarrow \infty}\left[\frac{\exp \left(\mathcal{U B}_{n}\right)-\exp \left(\mathcal{R}_{(n)}\right)}{\exp \left(\mathcal{U} \mathcal{B}_{(n-1)}\right)-\exp \left(\mathcal{R}_{(n-1)}\right)}\right] \rightarrow \infty
$$

Consequently, by the definition of the $\mathrm{d}^{\prime}$ Alemberts Ratio Test, $\exp \left(\mathcal{U} \mathcal{B}_{n}\right)-\exp \left(\mathcal{R}_{(n)}\right)$ diverges as $\mathcal{R}_{n}$ increases unboundedly. This in turn implies that,

$$
\begin{aligned}
& \mathcal{U B}_{n}>\mathcal{R}_{(n)} \\
& \text { UB Ratio Test }
\end{aligned}
$$

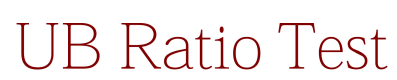

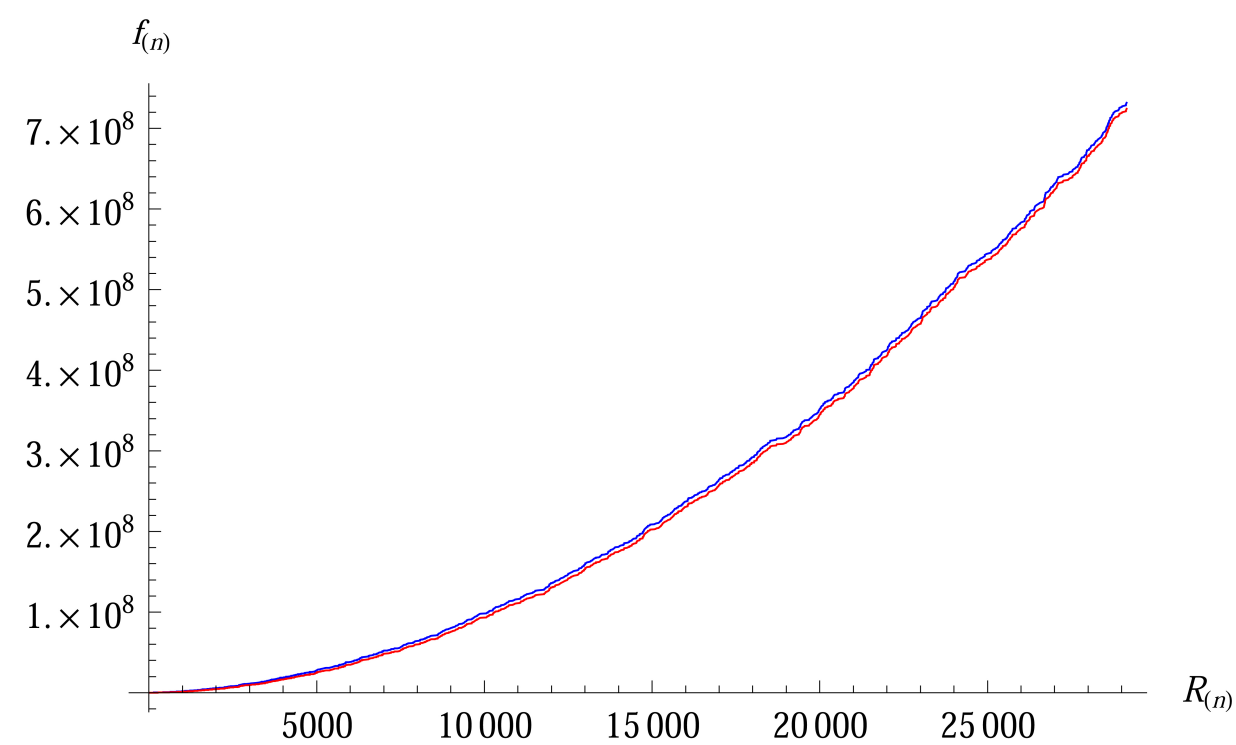

Figure 13. The drawing shows the ratio 5.15 (Blue) and the function 5.16 (Red). The Figure is drawn at every $\mathcal{R}_{(n)}$ in the range $11 \leq \mathcal{R}_{(n)} \leq 29147$

The upper bound on $\mathcal{R}_{n}$ as given by Laishram's Theorem (as quoted by Sondow et all [17]) in terms of the prime $p_{(3 n)}$ is given by:

Theorem 5.3 (Laishram).

For all $n \geq 1$, we have $\mathcal{R}_{n}<p_{(3 n)}$.

Sondow et al [17] further sharpens this result obtaining:

Theorem 5.4 (Sondow et al).

The maximum value of $\mathcal{R}_{n} / p_{(3 n)}$ is:

$$
\max _{n \geq 1} \frac{\mathcal{R}_{n}}{p_{(3 n)}}=\frac{\mathcal{R}_{5}}{p_{(15)}}=\frac{41}{47} \approx 0.8723
$$



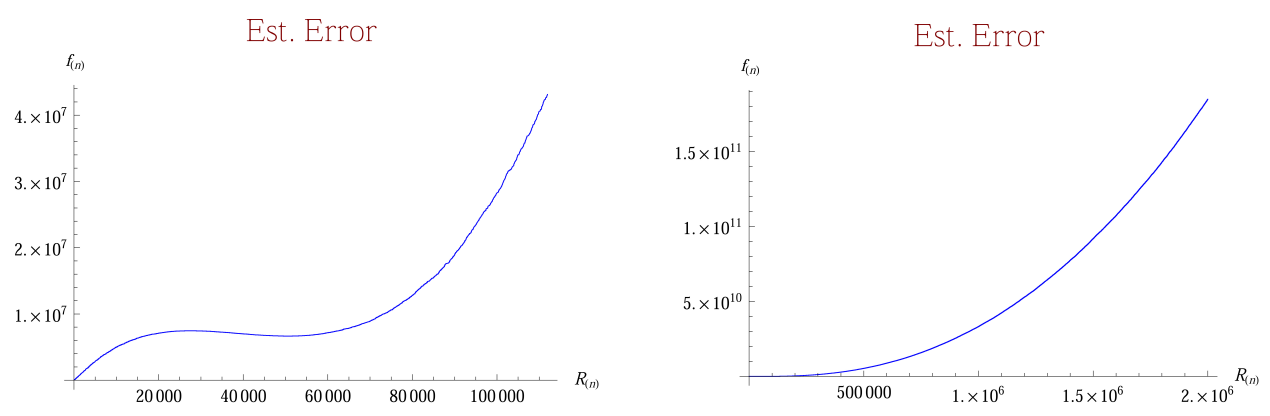

Figure 14. The drawing shows the estimation error made by the function 5.16. The L.H. figure is drawn at every $\mathcal{R}_{n}$ in the range $11 \leq \mathcal{R}_{n} \leq 111953$, while R.H. figure is drawn at every $\mathcal{R}_{n}$ in the range $11 \leq \mathcal{R}_{n} \leq 1999993$.

\section{Bounds on $R_{n}$}

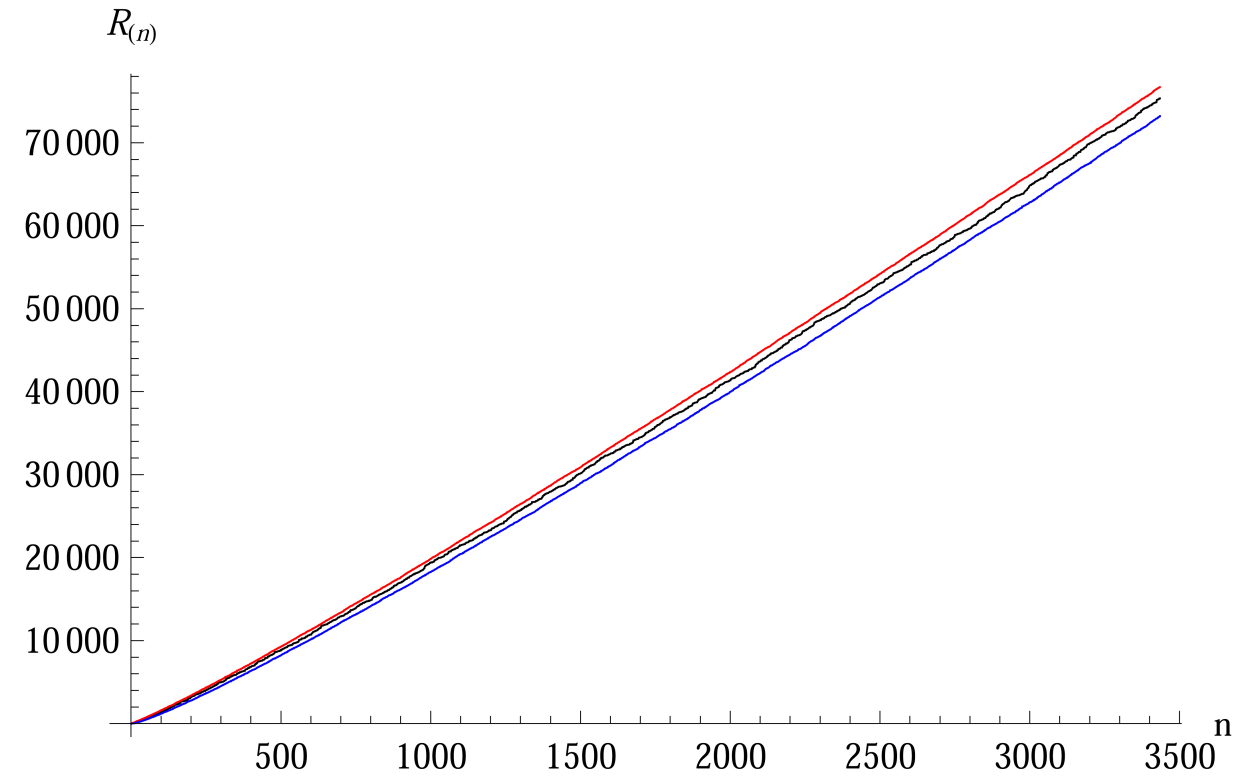

Figure 15. The drawing shows the Upper (Red) and Lower (Blue) bounds on $\mathcal{R}_{n}$ (Black). The Figure is drawn at every $n$ in the range $1 \leq n \leq 3435$.

In this paper we introduce an enhanced version of the upper and the lower bounds in terms of $p_{(2 n)}, p_{(3 n)}$ on the Ramanujan Interval:

Theorem 5.5 (Bounds on $\mathcal{R}_{n}$ in terms of $\left.p_{(2 n)}, p_{(3 n)}\right)$. by:

The bounds on $\mathcal{R}_{n}$ for all $n \in \mathbb{N} \mid n \geq 1$, in terms of $p_{(2 n)}$ and $p_{(3 n)}$ are given

$$
\frac{2353}{2000}\left(p_{(2 n)}\right)^{\alpha}-5 \sqrt{p_{(2 n)}}<\mathcal{R}_{n}<(\log (2))\left(p_{(3 n)}\right)+5 \sqrt{p_{(3 n)}}
$$



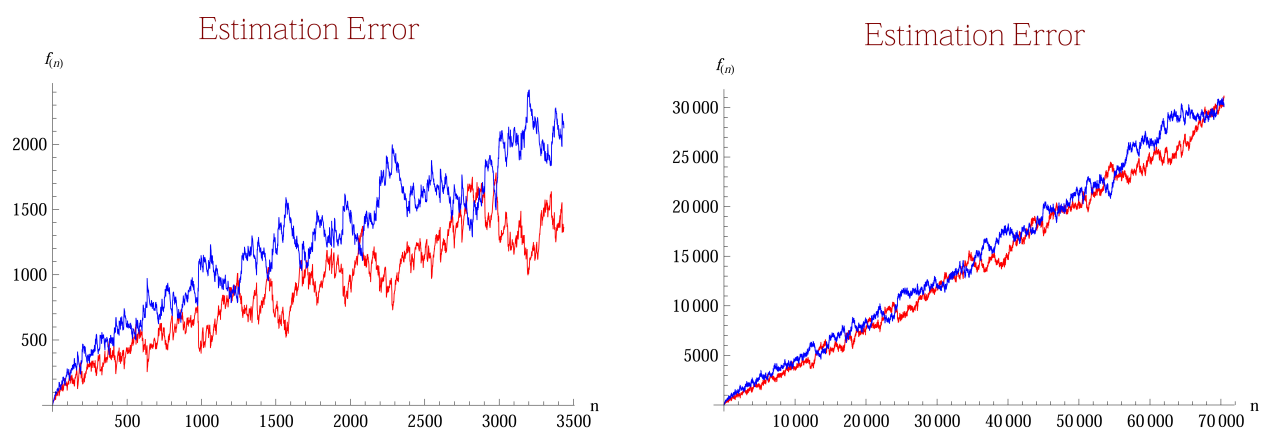

FIguRE 16. The drawing shows the estimation error made by the Upper (Red) and Lower (Blue) Bounds on $\mathcal{R}_{n}$. The R.H. figure is drawn at every $n \in \mathbb{N}$ in the range $1 \leq n \leq 70435$.

With the exponent $\alpha$ given by $\frac{9921}{10000}$.

The proof is limited to the proof of the Lower Bound only. The proof of the Upper Bound is left at the discretion of the reader.

Proof.

We begin with:

$$
\mathcal{R}_{n} \geq \mathcal{L} \mathcal{B}_{n}
$$

Suppose that Theorem 5.5 is false for some $\mathcal{R}_{n} \in \mathbb{N} \mid \mathcal{R}_{n} \geq 151$. In accordance with the hypothesis this implies that:

$$
\exp \left(\mathcal{R}_{n}\right)-\exp \left(\mathcal{L B}_{n}\right)<0
$$

However, at $\mathcal{R}_{n}=151$ the difference 5.20 attains approximately $3.7885 \times 10^{65}$ and further increases exponentially. Therefore, we apply the d'Alemberts Ratio Test. Define a sequence for all prime numbers $\mathcal{R}_{(n-1)}, \mathcal{R}_{n} \in \mathbb{N} \mid \mathcal{R}_{n} \geq 151$ :

Definition 5.6. $a_{(n)}=\frac{\exp \left(\mathcal{R}_{n}\right)-\exp \left(\mathcal{L B}_{n}\right)}{\exp \left(\mathcal{R}_{(n-1)}\right)-\exp \left(\mathcal{L} \mathcal{B}_{(n-1)}\right)}$

Remark 5.3. The sequence $a_{(n)}$ given by the Definition 5.6, has the least value at the Ramanujan twin primes, since the difference $\mathcal{R}_{(n)}-\mathcal{R}_{(n-1)}=2$. Consequently, it is therefore both necessary and sufficient, to consider the sequence $a_{(n)}$ at the Ramanujan twin primes only, with $\mathcal{R}_{(n)}=6 i+7 \mid i \in \mathbb{N}, i \geq 24$.

\section{At the Ramanujan twin primes:}

$$
\exp \left(\mathcal{R}_{(n)}\right)=\exp \left(\mathcal{R}_{(n-1)}+2\right)=\exp \left(\mathcal{R}_{(n-1)}\right) \times \exp (2)
$$

Thus, at the Ramanujan twin primes the sequence $a_{(n)}$ equals:

$$
a_{(n)}=\frac{\exp \left(\mathcal{R}_{(n)}\right)-\exp \left(\mathcal{L B}_{n}\right)}{\exp \left(\mathcal{R}_{(n-1)}\right)-\exp \left(\mathcal{L B}_{(n-1)}\right)}=\exp (2) \times\left[\frac{\exp \left(\mathcal{R}_{(n-1)}\right)-\frac{\exp \left(\mathcal{L B} \mathcal{B}_{n}\right)}{\exp (2)}}{\exp \left(\mathcal{R}_{(n-1)}\right)-\exp \left(\mathcal{L B} \mathcal{B}_{(n-1)}\right)}\right]
$$


The bracketed expression on the RHS, at the Ramanujan twin primes approaches the limit:

$$
\lim _{n \rightarrow \infty}\left[\frac{\exp \left(\mathcal{R}_{(n-1)}\right)-\frac{\exp \left(\mathcal{L B}_{n}\right)}{\exp (2)}}{\exp \left(\mathcal{R}_{(n-1)}\right)-\exp \left(\mathcal{L} \mathcal{B}_{(n-1)}\right)}\right] \rightarrow 1
$$

Therefore, at the Ramanujan twin primes, the sequence $a_{(n)}$ must clearly approach the limit:

$$
\left.\lim _{n \rightarrow \infty} a_{(n)}=\lim _{n \rightarrow \infty}\left[\exp (2) \times\left[\frac{\exp \left(\mathcal{R}_{(n-1)}\right)-\frac{\exp \left(\mathcal{L} \mathcal{B}_{n}\right)}{\exp (2)}}{\exp \left(\mathcal{R}_{(n-1)}\right)-\exp (\mathcal{L B}(n-1)}\right)\right]\right] \rightarrow \exp (2)
$$

By the definition of the d'Alemberts Ratio Test therefore, the difference $\exp \left(\mathcal{R}_{(n)}\right)-$ $\exp \mathcal{L B}_{n}$, diverges as $\mathcal{R}_{(n)}$ increases unboundedly. Thus, it logically follows that at Ramanujan twin primes:

$$
\exp \left(\mathcal{L B}_{n}\right) \leq \exp \left(\mathcal{R}_{(n)}\right) \quad \forall \mathcal{R}_{(n)} \in \mathbb{N} \mid \mathcal{R}_{(n)} \geq 151
$$

Necessarily therefore, we have a contradiction to the initial hypothesis. Since at the Ramanujan twin primes the sequence $\exp \left(\mathcal{R}_{(n)}\right)-\exp \mathcal{L B}_{n}$ approaches:

$$
\left[\exp \left(\mathcal{R}_{(n)}\right)-\exp \left(\mathcal{L B}_{n}\right)\right] \sim \exp (2)\left[\exp \left(\mathcal{R}_{(n-1)}\right)-\exp \left(\mathcal{L B}_{(n-1)}\right)\right]
$$

Rearranging the above, we obtain that at the Ramanujan twin primes $\exp \mathcal{L} \mathcal{B}_{n}$ approaches:

$$
\exp \left(\mathcal{L B}_{n}\right) \sim \exp \left(\mathcal{R}_{(n)}\right)-\exp (2)\left[\exp \left(\mathcal{R}_{(n-1)}\right)-\exp \left(\mathcal{L B}_{(n-1)}\right)\right]
$$

This in turn implies that a strict inequality holds:

$$
\exp \left(\mathcal{L B}_{n}\right)<\exp \left(\mathcal{R}_{(n)}\right)
$$

Since increasing the gap between the consecutive primes has the effect of exponentially increasing the value that the sequence $a_{(n)}$ attains, therefore this result holds for all $\mathcal{R}_{(n)} \in \mathbb{N} \mid \mathcal{R}_{(n)} \geq 151$. By taking the logarithms of both sides, we obtain:

$$
\mathcal{L B}_{n}<\mathcal{R}_{(n)} \quad \forall \mathcal{R}_{(n)} \in \mathbb{N} \mid \mathcal{R}_{(n)} \geq 151
$$

Thus, Theorem 5.5 holds for all $\mathcal{R}_{(n)} \in \mathbb{N} \mid \mathcal{R}_{(n)} \geq 151$. Direct computation verifies that Theorem 5.5 holds for all $\mathcal{R}_{(n)} \in \mathbb{N} \mid 2 \leq \mathcal{R}_{(n)} \leq 151$. Please refer to Figures 15 and 16. Therefore, Theorem 5.5 holds as stated:

$$
\mathcal{L B}_{n}<\mathcal{R}_{(n)} \quad \forall n \in \mathbb{N} \mid n \geq 1
$$

Concluding the proof of Theorem 5.5. 


\section{REFERENCES}

[1] Paul Erdös, On the difference of consecutive primes, Quarterly Journal Of Mathematics (1935).

[2] Paul Erdös and E.G. Strauss, Remarks on the differences between consecutive primes, Elem. Math. 35 (1980), 115-118.

[3] Jan Feliksiak, The elementary proof of the Riemann's Hypothesis, MDPI, AG, https://doi.org/10.20944/preprints202006.0365.v2 (2020).

[4] - The maximal prime gaps Supremum and the Firoozbakht's Hypothesis No 30, MDPI, AG, https://doi.org/10.20944/preprints202006.0366.v1 (2020).

[5] L Maximal prime gaps bounds, ScienceOpen, Inc, https://doi.org/10.14293/S21991006.1.SOR-.PPWVKRR.v1 (2021).

[6] The Binary Goldbach Conjecture, ScienceOpen, Inc, https://doi.org/10.14293/S2199-1006.1.SOR-.PPIU46T.v1 (2021).

[7] G.H. Hardy and E.M. Wright, An introduction to the theory of numbers, Oxford University Press, London, 1968.

[8] D.R. Heath-Brown, Differences between consecutive primes, Jahresber. Deutsch. Mathem. Ver. 90 (1988), 71-89.

[9] Edmund Landau, Vorlesungen über Zahlentheorie, Vol. II, S. Hirzel, Leipzig, 1927.

[10] János Pintz, Very large gaps between consecutive primes, Journal Of Number Theory (1997).

[11] Robert A. Rankin, The difference between consecutive prime numbers, Journal London Mathematical Society (1938).

[12] Atle Selberg, On the normal density of primes in small intervals and the difference between consecutive primes, Arch. Mathem. B 47 (1943), 87-105.

[13] _ An elementary proof of the prime number theorem, Ann. Math. 50 (1949), 305-313.

[14] Daniel Shanks, On maximal gaps between successive primes, Math. Comp. 18 (1964), 646651.

[15] Tomas Oliveira e Silva, Gaps between consecutive primes, 2006. www.ieeta.pt/ tos/gaps.html.

[16] Jonathan Sondow, Ramanujan primes and Bertrand's postulate, American Mathematical Monthly 116 (2009), 630-635.

[17] Jonathan Sondow, John Nicholson W., and Tony Noe D., Ramanujan primes: Bounds, Runs, Twins and Gaps, Journal of Integer Sequences 18 (2011).

[18] K. Soundararajan, Small gaps between prime numbers: the work of Goldston-Pintz-Yildirim, Bulletin of the American Mathematical Society (2007).

[19] Anitha Srinivasan and John W. Nicholson, An improved bound for Ramanujan Primes, Integers 15 (2015).

[20] E. Westzynthius, Über die Differenzen Verteilung der Zahlen die zu den $n$ ersten Primzahlen teilerfremd sind, Comm. Phys. Math. Soc. Sci. Fenn. 5 (1931), 1-37. 


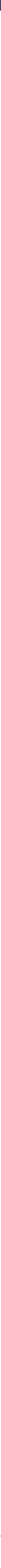

$\varepsilon$

욜

$\bar{x}$

气

r

동 